\title{
FLUORINE PRODUCTION FROM AGB AND WR STARS
}

\author{
NAMI MOWLAVI AND GEORGES MEYNET \\ Geneva Observatory \\ CH-1290 Sauverny, Switzerland
}

\begin{abstract}
The role of Wolf-Rayet and asymptotic giant branch stars in the production of the Solar System fluorine abundance is analyzed. It is shown that both these stars can be important sources of galactic fluorine. However, the uncertainties affecting the predictions, especially those from asymptotic giant branch stars, do not allow to give quantitative figures yet.
\end{abstract}

\section{Introduction}

The origin of the Solar System fluorine was unknown until ten years ago. In 1989, [2] suggested that this element can be produced in helium-burning environment in the presence of neutrons. The nucleosynthesis path follows ${ }^{14} \mathrm{~N}(\alpha, \gamma){ }^{18} \mathrm{~F}\left(\beta^{+}\right){ }^{18} \mathrm{O}(\mathrm{p}, \alpha){ }^{15} \mathrm{~N}(\alpha, \gamma){ }^{19} \mathrm{~F}$. The required protons would be provided by ${ }^{14} \mathrm{~N}(\mathrm{n}, \mathrm{p}){ }^{14} \mathrm{C}$, with neutrons liberated from ${ }^{13} \mathrm{C}(\alpha, \mathrm{n}){ }^{16} \mathrm{O}$. Both ${ }^{13} \mathrm{C}$ and ${ }^{14} \mathrm{~N}$ can result from the ashes of $\mathrm{H}$-burning by the $\mathrm{CNO}$ cycles.

Two stellar sites have been proposed as candidates for the fluorine nucleosynthesis in He-burning environment. The first one is asymptotic giant branch (AGB) stars. The ${ }^{19} \mathrm{~F}$ production in those stars is strongly suggested by observations of fluorine overabundances in red giant $\mathrm{S}$ and $\mathrm{C}$ stars as compared to normal giants ([4]). Current stellar evolutionary models confirm the operation of the F-nucleosynthesis path in those stars, but fail to reproduce the observed overabundances $([1,9]$; see also [8] in this volume). A source of primary ${ }^{13} \mathrm{C}$ is required, which is difficult to obtain in $\mathrm{AGB}$ models (but see [3]). Predictions of the fluorine yield from AGB stars suffer thus from the shortcomings affecting AGB models (see [8] in this volume).

The second stellar site suggested for fluorine production in He-burning environments is Wolf-Rayet (WR) stars $([6,7])$. Contrary to the AGB stars, no observation of fluorine in WR stars has been reported or even attempted yet. However, the nucleosynthesis of that element in those massive stars 
rests on the evolution of WR stars which are relatively easy to model. The fluorine yields from WR stars are thus subject to much less uncertainties than those from AGB stars.

The role of WR stars in the galactic evolution of fluorine, and in particular in the production of its Solar System abundance is recalled in Sect. 2. That of AGB stars is analyzed in Sect. 3.

\section{Galactic fluorine from WR stars}

While no observation of the F abundance at the surface of WR stars has been reported so far, massive star model predictions for that element can be used to estimate the contribution of WR stars to the galactic fluorine. This has been done by [7], the details of which are not repeated here. Let us recall that these authors arrive to the conclusion that WR stars alone can account for the solar ${ }^{19} \mathrm{~F}$.

\section{Galactic fluorine from AGB stars}

Let us get an estimation of the ${ }^{19} \mathrm{~F}$ contamination by AGB stars to the Solar System fluorine abundance. For that purpose, we consider here that only AGB stars affect the galactic ${ }^{19} \mathrm{~F}$ budget, all other possible production or destruction sites being neglected. We make use of a very simple galactic chemical evolution model with the approximations of a closed box and instantaneous recycling. The procedure and notations are described in [7].

Because no fluorine yields are provided by AGB models, we evaluate them from the observed overabundances. If we consider a mean fluorine overabundance of $\left[{ }^{19} \mathrm{~F} /{ }^{16} \mathrm{O}\right] \equiv\left[\log { }^{19} \mathrm{~F} /{ }^{16} \mathrm{O}\right]-\left[\log { }^{19} \mathrm{~F} /{ }^{16} \mathrm{O}\right]_{\odot}$ in AGB stars, independent of stellar mass and metallicity (no observation of the $\mathrm{F}$ abundance in low-metallicity stars is available yet), we have

$$
X_{19}^{s}-X_{19}^{0}=X_{19}^{0}\left(10\left[{ }^{[9} \mathrm{F} /{ }^{16} \mathrm{O}\right]-1\right)
$$

where $X_{19}^{0}$ is the initial stellar fluorine mass fraction, and $X_{19}^{s}$ that at the surface of AGB stars.

Assuming, for simplification, that all AGB stars end as $0.6 \mathrm{M}_{\odot}$ white dwarfs, the yields from AGB stars of given initial mass $M_{i}$ are (Eq. 1 of [7])

$$
p_{19}^{A G B}\left(M_{i}\right)=\frac{M_{i}-0.6}{M_{i}} X_{19}^{0}\left(10^{\left[{ }^{19} \mathrm{~F} /{ }^{16} \mathrm{O}\right]}-1\right) .
$$

In order to derive the net ${ }^{19} \mathrm{~F}$ yield $y_{19}$ of a stellar generation, we use an initial mass function $\phi\left(M_{i}\right)=M_{i}^{-2.7} / \psi$ (the exponent being taken from [5]) with a star formation rate $\psi=10 \mathrm{M}_{\odot} \mathrm{pc}^{-2} \mathrm{Gyr}^{-1}$, and consider that only 
AGB stars with $1 \leq M_{i} \leq 4$ contribute to the interstellar ${ }^{19} \mathrm{~F}$ enrichment (stars with $M<1$ eject their envelope before entering the thermal-pulse AGB phase, while hot bottom burning would prevent AGB stars with $M>$ 4 to be efficient fluorine producers). This leads to

$$
\begin{aligned}
y_{19} & =\frac{1}{1-R} \int_{1}^{4} M_{i} p_{19}^{A G B}\left(M_{i}\right) \phi\left(M_{i}\right) \mathrm{d} M_{i} \\
& \simeq \frac{0.05}{1-R} X_{19}^{0}\left(10^{\left[{ }^{19} \mathrm{~F} /{ }^{16} \mathrm{O}\right]}-1\right)
\end{aligned}
$$

where $\mathrm{R}$ is the 'returned fraction' (see [10]).

Taking $R \simeq 0.17([10])$ and $\left[{ }^{19} \mathrm{~F} /{ }^{16} \mathrm{O}\right] \sim 0.5$, the ${ }^{19} \mathrm{~F}$ mass fraction $X_{19, \odot}$ at the time $t_{\odot}$ of Solar System formation is given by

$$
\begin{aligned}
X_{19, \odot} & =y_{19} \ln \left[\frac{1}{\sigma\left(t_{\odot}\right)}\right] \\
& \simeq 0.25 X_{19}^{0} .
\end{aligned}
$$

AGB stars would thus contribute to as much as $25 \%$ of the Solar System fluorine. This is only a very rough estimate, the most criticable assumptions being instantaneous recycling and the independency of fluorine production with stellar metallicity. It does show, however, that AGB stars are potentially important contributors to the Galactic fluorine.

\section{References}

1. Forestini M., Goriely S., Jorissen A., Arnould M.: 1992, A\&A 261, 157

2. Goriely S., Jorissen A., Arnould M.: 1989, in Proc. 5th Workshop in Nuclear Astrophysics; MPA/P1, eds. W. Hillebrandt and E. Müller, Garching, Max Planck Institut für Astrophysik, p. 60

3. Herwig F., Blöcker T., Xchönberner D., El Eid M.: 1997, A\&A 324, L81

4. Jorissen A., Smith V.V., Lambert D.L.: 1992, A\&A 261, 164

5. Kroupa P., Tout C., Gilmore G., 1993, MNRAS 262, 545

6. Meynet G., Arnould M.: 1993, in Int. Symp. on Nuclear Astrophysics, Karlsruhe, IOP Publishing Ltd

7. Meynet G., Arnould M.: 1996, in 32nd Liège Int. Astroph. Coll., eds. Noels A., Fraipont-Caro D., Gabriel M., Grevesse N., Demarque P., pp 89-95

8. ${ }^{1}$ Mowlavi N., this volume

9. ${ }^{1}$ Mowlavi N., Jorissen A., Arnould M: 1996, A\&A 311, 803

10. Tinsley B.: 1980, Fundamentals of Cosmic Physics 5, 287

${ }^{1}$ postscript files available by anonymous ftp on 'obsftp.unige.ch' in 'pub/mowlavi', or in 'http://obswww.unige.ch/ mowlavi/publications/publications.html 